Proc. of the X Int. Conf. - Ion Implantation and other Applications of Ions and Electrons, Kazimierz Dolny 2014

\title{
Temperature Dependent Variations of Properties of Polymer-Like Carbon Coatings Treated with High Energy Electrons
}

\author{
R. Plaipaité-Nalivaiko*, L. Vigricaitė, D. Adlienė and Ž. Rutkūnienė \\ Kaunas University of Technology, Physics Department, Studentų str. 50, LT-51368, Lithuania
}

\begin{abstract}
Polymer-like carbon coatings can be used for the protection of micro-electromechanical devices functioning in chemically or radiation harsh environment due to their beneficial properties. Properties of carbon coatings depend on the method of synthesis, initial gas mixture, admixture of additives, temperature and other technological parameters. Modification of carbon structures is possible applying high energy electrons. Coating mechanical properties become almost stable after their pretreatment with some doses of high energy electrons. Polymer-like carbon coatings were synthesized from acetylene gas plasma on $n$-type silicon substrates in the RF plasmotron system at temperatures in the range 293-673 K using plasma enhanced chemical vapor deposition method. Series of samples were prepared and irradiated with high energy $(6 \mathrm{MeV})$ electrons in a medical linear accelerator. The ellipsometric method was used for estimation of some optical parameters and thickness of synthesized coatings. Structural changes of polymer-like carbon coatings were evaluated using the Raman spectroscopy and surface morphology was assessed by atomic force microscopy. Electron beam induced structural changes in the coatings were analyzed using the obtained results. The relations between the synthesis temperature and samples structure and surface morphology are discussed.
\end{abstract}

DOI: 10.12693 /APhysPolA.128.915

PACS: 61.80.Fe, 68.37.Ps, 78.30.Jw, 78.70.--g

\section{Introduction}

Carbon coatings attract attention due to their unique chemical and physical properties $[1,2]$. As regards deposition conditions and technological parameters carbon coatings can be classified as polymer-like, diamond-like, or graphite-like based on the main binding framework. Carbon coatings can exist in different kinds of matrices and are usually doped with a large amount of hydrogen thereby making the materials even more diverse. In some cases hydrogen content in a film can reach up to 50 at.\%. These coatings consist of an amorphous matrix with carbon clusters and crystallites in it [3]. Mechanical properties of a-C:H films are defined by $s p^{3}$ bonds, while optical and electrical properties depend upon $s p^{2}$ bonds. Hydrogen can affect mechanical properties of the coatings due to the decreased content of $s p^{3}$ bonds [1-3]. Lowdensity, soft films with large hydrogen fraction in it are called polymer-like carbon (PLC) coatings [4]. Due to the unique dielectric, optical and mechanical properties PLC coatings are promising material for the protection of micro-electromechanical (MEM) devices functioning in chemically or radiation harsh environment. PLC coatings are characterized by a wide optical band gap (1.8$4 \mathrm{eV}$ ) [5]. Choosing a deposition method, varying initial gas mixture content, admixing additives, changing fabrication conditions or technological parameters, it is possible to deposit PLC coatings with large hydrogen and $s p^{3}$

*corresponding author; e-mail: rita.plaipaite@gmail.com bond contents, low density and exhibit special optical absorption, intense photoluminescence and electron affinity. A special attention should be paid to the coating deposition temperature since it affects structural and some mechanical properties of synthesized coatings [1, 6, 7].

Irradiation of carbon coatings to high energy electrons is one of the possibilities applied for modification of their mechanical properties since electron irradiation causes structural changes in irradiated structures that, in turn, are directly linked to the coating surface morphology changes and influence mechanical properties of carbon coatings.

Modification of a-C:H coatings using high energy photons and electrons was also observed and investigated in our previous papers $[1,8,9]$. The main purpose of this paper was to evaluate and discuss structural changes and properties modification of PLC coatings RF plasma produced at different deposition temperatures after their exposure to high energy electrons.

\section{Experimental}

PLC coatings were synthesized by the plasma enhanced chemical vapor deposition (PECVD) method in a $\mathrm{RF}(13.56 \mathrm{MHz})$ dual-plasma reactor on $n$-type silicon (100) substrates. Prior to the deposition silicon substrates were cleaned and etched in acetone. The precleaned substrates were pre-treated in $\mathrm{Ar}^{+}$plasma in order to achieve an oxygen free surface and a buffer layer and to enhance coating adhesion.

Deposition of carbon coatings was performed at different temperatures ranging from 298 to $673 \mathrm{~K}$ using acetylene $\left(\mathrm{C}_{2} \mathrm{H}_{2}\right)$ as a precursor. The deposition time of PLC coatings was $5 \mathrm{~min}$. The deposition pressure of $33 \mathrm{~Pa}$ was 
maintained during the entire deposition process. 5 series of samples were prepared and evaluated. The index TA was assigned to the "as-prepared" samples and index TE - to the electron beam irradiated samples.

Irradiation of samples to high energy (6 MeV) electrons was performed in a medical linear accelerator Clinac2100C (VARIAN) at the dose rate $3 \mathrm{~Gy} / \mathrm{min}$. The maximal irradiation dose was 3 Gy. The structure of as-prepared and irradiated PLC coatings was ex situ characterized using the Raman spectroscopy (RS). The Raman spectra of the samples were obtained in a range of $1000-2000 \mathrm{~cm}^{-1}$ using the Yvon Jobin spectrometer with a Spectra Physics Nd:YAG laser $(\lambda=$ $532.3 \mathrm{~nm}, 50 \mathrm{~mW}$ at a $0.3 \mathrm{~mm}$ spot size). The overlapped background corrected Raman spectral bands were fitted with the multi-Gaussian line-shapes, using the Least Square Fitting software.

Surface morphology of PLC coatings was characterized by atomic force microscope (AFM NT-206) using V-shaped ULTRASHARP Si cantilever (force constant $1.5 \mathrm{~N} \mathrm{~m}^{-1}$ ). The measurements were performed using a tapping mode. The suitable software was used for the evaluation of the roughness parameters of coatings within the scanned area of $3 \mu \mathrm{m} \times 3 \mu \mathrm{m}$. The sample thickness, refractive index and extinction coefficient were estimated using the Laser ellipsometer Gaertner 117 with a $\mathrm{He}-\mathrm{Ne}$ laser $(\lambda=632.8 \mathrm{~nm})$.

\section{Results and discussion}

\subsection{Ellipsometric measurements}

The summary of the main characteristics of asprepared PLC coatings obtained from the ellipsometric measurements is given in the Table. Ellipsometric results show the decreasing thickness of the samples with the increase in deposition temperature. There is no wellestablished tendency observed between the refractive index or the extinction coefficient and the temperature since both parameters strongly depend on the formed carbon coating structure and hydrogen content in it as it was shown analyzing the Raman spectra of the samples.

TABLE

Characteristics of PLC coatings.

\begin{tabular}{l|c|c|c|c|c|c}
\hline \hline ID & $\begin{array}{c}\text { Precursor } \\
\text { gas }\end{array}$ & $\begin{array}{c}\text { Bias } \\
\text { voltage } \\
{[\mathrm{V}]}\end{array}$ & $\begin{array}{c}\text { Temp. } \\
{[\mathrm{K}]}\end{array}$ & $\begin{array}{c}\text { Refractive } \\
\text { index } \\
(n)\end{array}$ & $\begin{array}{c}\text { Extinction } \\
\text { coefficient } \\
(k)\end{array}$ & $\begin{array}{c}\text { Film } \\
\text { thickness } \\
{[\mathrm{nm}]}\end{array}$ \\
\hline TA-4 & & & 298 & 1.73 & 0.001 & 274 \\
TA-5 & acetylene & & 405 & 1.65 & 0.008 & 268 \\
TA-3 & 200 & 481 & 1.66 & 0.012 & 251 \\
TA-6 & $\left(\mathrm{C}_{2} \mathrm{H}_{2}\right)$ & & 575 & 1.69 & 0.009 & 221 \\
TA-7 & & & 673 & 1.62 & 0.018 & 130
\end{tabular}

\subsection{Raman spectroscopy}

The Raman spectroscopy was used for the investigation of bonding structure of the fabricated coatings. Using this method it is possible to estimate the $s p^{3} / s p^{2}$ ratio in the investigated samples, which corresponds to the coating structural organization and hydrogen content in it. The bonding structure and hydrogen content are the main parameters influencing optical, electrical, and mechanical properties of carbon coatings.

The Raman spectra of some coatings before and after their irradiation with high energy electrons are presented in Fig. 1. Notwithstanding the fact that the structural organization level was different in the samples belonging to different series, according to [5], the samples can be classified as polymer-like coatings. In each form of carbon coatings, RS is determined by the vibrations of any $s p^{2}$ sites because of the resonant enhancement of their scattering. The main role, defining the type of carbon coating, is played by the $G$ peak at $1500-1630 \mathrm{~cm}^{-1}$ which arises from the bond stretching motion of pairs of $s p^{2} \mathrm{C}$ atoms in aromatic rings or olefinic chains [4, 9], and the $D$ peak, which becomes active only in the presence of disorder due to the breathing modes of rings.

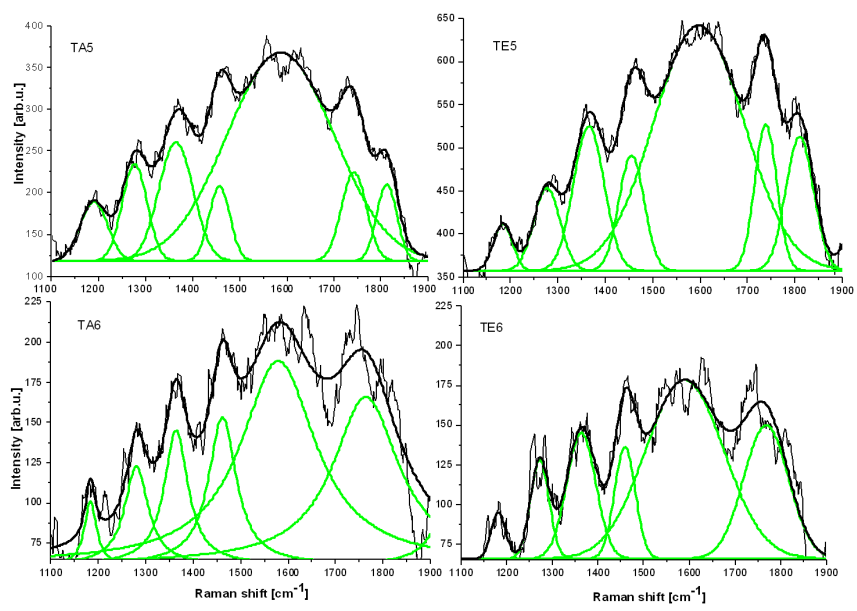

Fig. 1. Raman spectra of as-prepared (TA-5 and TA$6)$ and irradiated (TE-5 and TE-6) samples.

It is evident that the Raman spectra of the samples (Fig. 1) contain some additional intrinsic peaks indicating a more complicated structure of carbon coatings. The main Raman peaks of TA-5 sample were obtained at $1364 \mathrm{~cm}^{-1}(D$ peak $)$ and at $1586 \mathrm{~cm}^{-1}$ ( $G$ peak $)$. Both peaks change their shape, intensity and position after irradiation with high energy electrons. The $D$ and $G$ peaks become more intensive and shift to the higher wave number $\left(1597 \mathrm{~cm}^{-1}\right)$. The full width at halfmaxima (FWHM) of $D$ and $G$ peaks is narrowed after the modification. The integral intensity ratio $I_{D} / I_{G}$ increases from 0.57 to 0.59 . According to [3] there can be some reasons for the shape of the spectrum and small FWHM values, i.e.: changes of bonding structure in the irradiated coating (increased number of $s p^{2}$ sites), radiation induced stress and formation of clusters in the PLC coating, their size and distribution. A similar tendency for Raman peak shifting was observed analyzing the TA-6 sample, however, the overall relative intensity of irradiated coating was lower. After irradiation $D$ peak remains at the same position $-1363 \mathrm{~cm}^{-1}$, however, the 
intensity of this peak slightly increases. The increased intensity of $D$ peak could be explained by the conversion of $s p^{3}$ bonds to $s p^{2}$ bonds, desorption of hydrogen and formation of graphite nanoclusters due to radiation induced scission and crosslinking processes in the polymeric structure. $G$ peak of irradiated sample (TE-6) shifts from $1579 \mathrm{~cm}^{-1}$ to $1589 \mathrm{~cm}^{-1}$ and becomes broader. It should be stressed that the FWHM values of both peaks are higher after the irradiation and the $I_{D} / I_{G}$ ratio increases from 0.62 to 0.71 . Therefore it is possible to suggest that this coating became more graphite-like after its irradiation with high energy electrons.

In parallel some intrinsic peaks are observed for our samples: the peak at about $1190 \mathrm{~cm}^{-1}$ can be attributed to high $s p^{3}$ content or nanocrystalline phase of diamond; peak at about $1275 \mathrm{~cm}^{-1}$ can be assigned to $\mathrm{C}-\mathrm{O}-\mathrm{C}$ asymmetric stretch; the semicircle ring stretch vibration of benzene or condensed benzene rings [3] are responsible for peak at $\approx 1460 \mathrm{~cm}^{-1}$; peaks at about $1637 \mathrm{~cm}^{-1}$ and $1750 \mathrm{~cm}^{-1}$ can be attributed to $\mathrm{C}=\mathrm{C}$ stretching vibration. The peak $\approx 1812 \mathrm{~cm}^{-1}$ (for TA-5 and TE-5 only) can be assigned to $\mathrm{C}=\mathrm{O}$ vibration mode.

\subsection{Atomic force microscopy}

The surface conditions play an important role applying PLC as protective coatings. Due to this, surface morphology of experimental samples was examined using $\mathrm{AFM}$ and the roughness values were determined. 3D topographic scans of the samples before and after their modification by $6 \mathrm{MeV}$ electron beam are presented in Fig. 2. Each scan represents a $3 \mu \mathrm{m} \times 3 \mu \mathrm{m}$ lateral area.
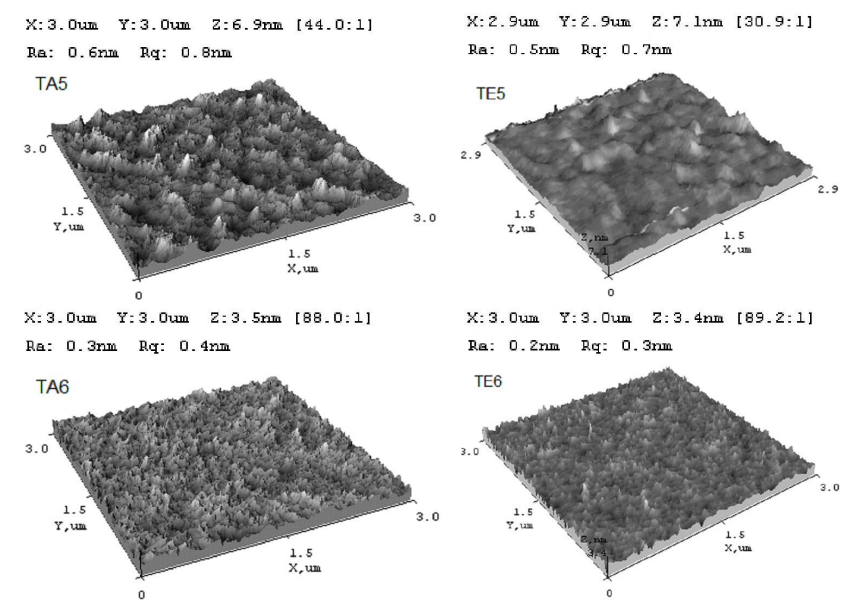

Fig. 2. 3-dimensional AFM images of as-prepared (left) and irradiated (right) samples.

It is evident that the structure of carbon coating produced at higher temperatures ( $575 \mathrm{~K}$ for TA-6) is more polymer-like and smoother as compared to the samples produced at lower temperatures (405 K for TA-5). With reference to other publications electron bombardment of samples is responsible for hydrogen evaporation [8] and radiation induced polymerization processes [7]. Surface confined polymerization can significantly change surface morphology and make rough surfaces smoother as it is observed in the case of irradiated coatings. It should be noted that the surface roughness of irradiated coatings decreases in accordance to rearrangements in the structure of the coatings that, in turn, are dependent on the electron beam energy and transferred dose.

\section{Conclusions}

PLC coatings were produced using the PECVD method from $\mathrm{C}_{2} \mathrm{H}_{2}$ varying deposition temperature in the range from 293 to $673 \mathrm{~K}$. It was found that the RS of the fabricated PLC coatings in parallel with the $D$ and $G$ peaks is characterized by a number of intrinsic peaks, assigned to $\mathrm{C}=\mathrm{C}, \mathrm{C}=\mathrm{O}$ stretching vibrations, $\mathrm{C}-\mathrm{O}-\mathrm{C}$ asymmetric stretch, indicating also possible presence of nanocrystalline phase of diamond and high $s p^{3}$ content of disordered structure. The increase of the bonding ratio $s p^{2} / s p^{3}$ was observed with the increased deposition temperature. This phenomenon might be explained by the fact that the $s p^{2}$ sites are induced varying coating deposition temperature or bombarding the samples with high energy $(6 \mathrm{MeV})$ electrons. High energy electrons are able to reconstruct a network structure of sample due to radiation induced polymerization processes (chain scission and crosslinking) that result in clusterization and graphitization processes. The occurrence of clusterization and graphitization processes as well as changes in surface morphology in the PLC coatings can be also influenced by deposition temperature as it was proved exploring the AFM measurements. The clusters, that can be in the form of olefinic chains or aromatic rings, are a good indicator investigating optical and other physical properties of PLC coatings.

\section{Acknowledgments}

This investigation was performed under the support of the European Social Fund Agency (project No. VP1-3.1ŠMM-08-K-01-013).

\section{References}

[1] M. Šniurevičùè, J. Laurikaitiee, D. Adlieè, L. Augulis, Ž. Rutkūnienè, A. Jotautis, Vacuum 83, 159 (2009).

[2] P. Chu, L. Li, Math. Chem. Phys. 96, 253 (2006).

[3] J. Schwan, S. Ulrich, V. Batori, H. Ehrhardt, S. Silva, J. Appl. Phys. 80, 440 (1996).

[4] A. Ferrari, J. Robertson, Phys. Rev. B 61, 14096 (2000).

[5] J. Robertson, Mater. Sci. Eng. R 37, 129 (2002).

[6] D. Adlienè, J. Laurikaitienè, S. Tamulevičius, Nucl. Instrum. Methods Phys. Res. B 266, 2788 (2008).

[7] R. Nathawat, A. Kumar, V. Kulshrestha, M. Singh, D.M. Phase, Y.K. Vijay, Appl. Surf. Sci. 253, 5985 (2007).

[8] R. Plaipaitè-Nalivaiko, D. Adlienè, G. Adlys, Acta Phys. Pol. A 123, 871 (2013).

[9] S. Rodil, A. Ferrari, J. Robertson, W. Milne, J. Appl. Phys. 89, 5425 (2001). 